EPJ Web of Conferences 60, 20001 (2013)

DOI: $10.1051 /$ epjconf/20136020001

(C) Owned by the authors, published by EDP Sciences, 2013

\title{
ATLAS measurements of Drell-Yan lepton-pair production as probes of the Standard Model
}

\author{
Manuella G. Vincter ${ }^{1, a}$ on behalf of the ATLAS Collaboration \\ ${ }^{1}$ Physics Department, Carleton University, Ottawa, ON, Canada
}

\begin{abstract}
Measurements of Drell-Yan lepton-pair production cross sections and associated angular correlations can provide unique insight into perturbative Quantum Chromodynamics in the absence of colour flow between initial and final states, the V-A nature of electroweak interactions, as well as enhance the knowledge of parton distribution functions in the proton. Drell-Yan production is also an important source of background for other Standard-Model processes such as Higgs-boson production as well as for new phenomena, particularly at high dilepton invariant mass, and so must be evaluated precisely at the Large Hadron Collider. The measurements presented in this note are based on approximately $5 \mathrm{fb}^{-1}$ of data collected at a centre-of-mass energy of $7 \mathrm{TeV}$ in 2011 with the ATLAS detector based at the CERN Laboratory.
\end{abstract}

\section{Introduction}

The results presented in this note are based on approximately $5 \mathrm{fb}^{-1}$ of data collected in 2011 at a centre-ofmass energy $\sqrt{s}=7 \mathrm{TeV}$ with the ATLAS [1] experiment based at the Large Hadron Collider (LHC) at the CERN Laboratory. Approximately three million DrellYan events [2-4] in the invariant-mass range of $66 \mathrm{GeV}$ to the $\mathrm{TeV}$ scale were recorded in both dielectron and dimuon final states, resulting in an impressive agreement between data and Standard-Model expectations over six orders of magnitudes, as is demonstrated in Figure 1. Such datasets enable detailed comparisons to next-to-next-toleading-order calculations, leading and next-to-leadingorder Monte Carlo (MC) generator predictions, and various parton distribution function parameterisations. DrellYan lepton-pair production is also an important source of background in many Standard-Model measurements as well as in searches for new physics, such as the production of the Z' particle with Standard-Model $Z$ couplings to fermions, which has been excluded by ATLAS at the $95 \%$ credibility level for masses below $2.86 \mathrm{TeV}$ [5]. Hence, it is of great interest to make precision measurements of the Drell-Yan process at LHC energies.

\section{Z-boson forward-backward asymmetry}

Due to the V-A nature of electroweak interactions, leptons produced in $Z$-boson decays are expected to display a forward-backward asymmetry with respect to the quark direction in the dilepton rest frame. This asymmetry $A_{\mathrm{FB}}$ defined by the ratio of the number $N$ of events

$$
A_{\mathrm{FB}}=\frac{N(\cos \theta \geq 0)-N(\cos \theta<0)}{N(\cos \theta \geq 0)+N(\cos \theta<0)}
$$

\footnotetext{
a e-mail: vincter@physics.carleton.ca
}

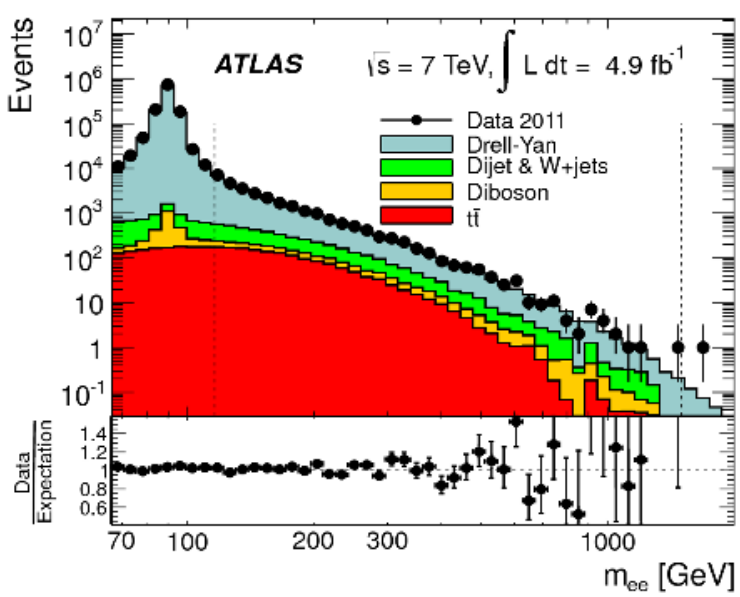

Figure 1. Drell-Yan invariant-mass distribution in the dielectron channel, comparing data to the sum of Standard-Model signal and background expectations [2].

is measured in ATLAS [3] using angles in the CollinsSoper frame [6], where the cosine of the decay angle is given as a function of the lepton momenta in the laboratory frame. The sign of $\cos \theta$ is determined from the direction of the incoming quark, which is ambiguous in $p p$ collisions, resulting in a dilution of the measured asymmetry. However, this dilution decreases as the boost of the dilepton system increases. The ATLAS experiment's unique ability to cleanly identify $Z$ bosons even beyond the pseudorapidity of its tracking system $(|\eta|<2.5)$ makes for a sensitivity to this measurement well beyond what might be expected at a $p p$ collider. These unique $Z$ bosons are acquired by requiring that one electron be identified in the central region $(\mathrm{C})$ of the detector, well covered by 


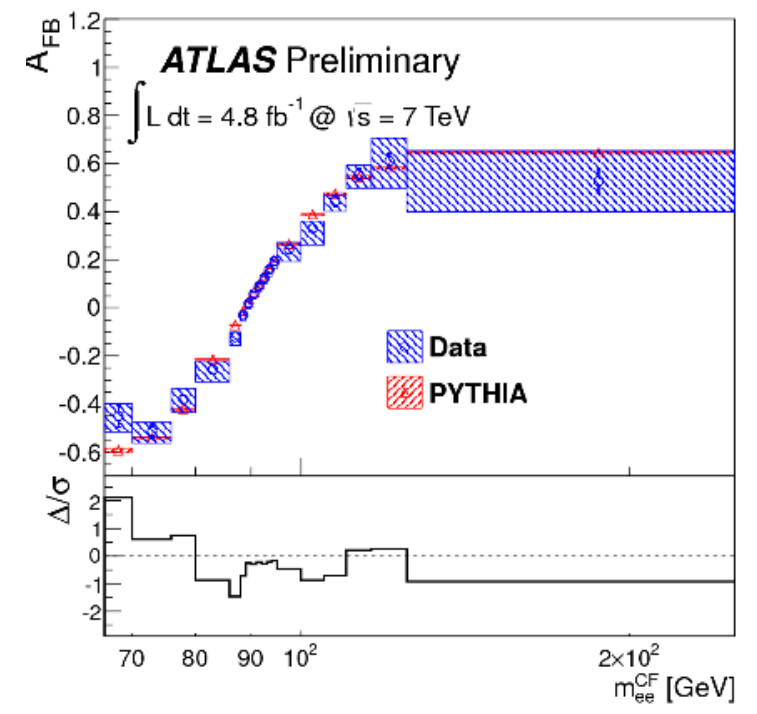

Figure 2. The fully unfolded forward-back asymmetry distribution for central-forward (CF) electrons [3]. The shaded regions represent the data (Monte Carlo) total (statistical) uncertainties. The ratio below shows the difference between data and MC divided by the quadratic sum of the data and MC uncertainties.

the ATLAS tracking system, and the other electron be in the forward (F) region $(2.5<|\eta|<4.9)$ where precise calorimeters are able to identify electromagnetic objects without the benefit of tracking, thereby enabling measurements of this asymmetry with significant precision in a region where the effect of the dilution factor is reduced. This asymmetry, fully unfolded for detector, QED radiative (to Born-level), and dilution effects is shown in Figure 2 for the central-forward (CF) combination of dielectrons in the invariant-mass region of $66-250 \mathrm{GeV}$, based on $4.8 \mathrm{fb}^{-1}$ of $p p$ collision data. Forward-backward asymmetries are also measured in the central region of ATLAS in both the dielectron and dimuon channels.

The asymmetry $A_{\mathrm{FB}}$ is sensitive to several StandardModel parameters, including the effective weak mixing angle $\sin ^{2} \theta_{\mathrm{W}}^{\text {eff }}$. The raw asymmetry distributions as measured in the dielectron and dimuon channels are used to extract $\sin ^{2} \theta_{\mathrm{W}}^{\text {eff }}$ by comparing the data to Monte Carlo predictions produced with various values of the weak mixing angle. Figure 3 shows the ATLAS results for dielectrons in the central-central (CC), central-forward (CF), and dimuon channels and compares the average of these results to world measurements made at the LHC and other colliders as well as to the results from a Particle Data Group (PDG) global fit [7]. The CF results are of better precision than either the electron $\mathrm{CC}$ or muon results, even with just $30 \%$ of either channel's statistics. The effective weak mixing angle results presented here are consistent with the PDG fit to world data, but still lack the precision achieved at lepton colliders.

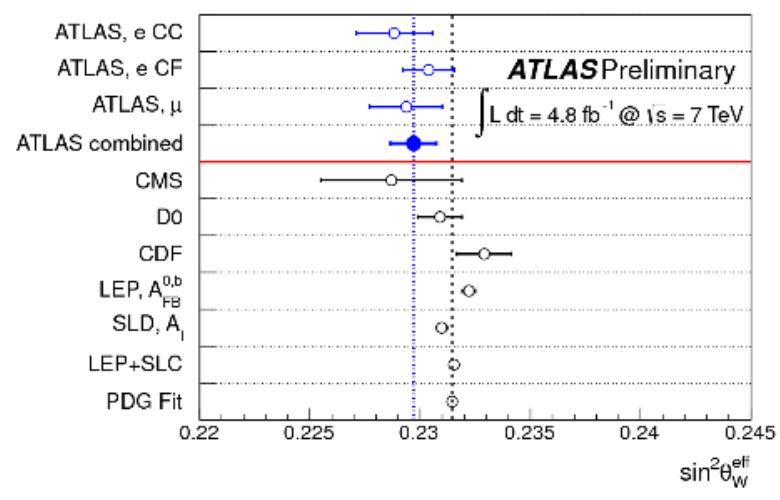

Figure 3. Comparison of $\sin ^{2} \theta_{\mathrm{W}}^{\text {eff }}$ measured in this analysis [3] with other results at the LHC and at other colliders. The vertical dotted line represents the average of the ATLAS measurements while the vertical dashed line represents the results of a PDG global fit [7].

\section{Measurement of $\phi_{\eta}^{*}$ in $Z$ production}

At the one-gluon emission level, $Z$-bosons produced in $p p$ collisions recoil against the hadronic products thereby giving transverse momentum to the $Z\left(p_{\mathrm{T}}^{\mathrm{Z}}\right)$. This implies that $p_{\mathrm{T}}^{\mathrm{Z}}$ carries information about the hard interaction and so is sensitive to the effects of the strong interaction.

An optimal experimental observable $\phi_{\eta}^{*}$ has been designed that probes the same physics information as $p_{\mathrm{T}}^{\mathrm{Z}}$ but with much better precision as it depends uniquely on the direction of the leptons, which is better measured than lepton momenta:

$$
\phi_{\eta}^{*} \equiv \tan [(\pi-\Delta \phi) / 2] \sin \left(\theta_{\eta}^{*}\right) .
$$

The variable $\Delta \phi$ is the azimuthal opening angle between the two leptons. The angle $\theta_{\eta}^{*}$ measures the scattering angle of the leptons with respect to the proton-beam direction in the rest frame of the dilepton system and is defined from the difference in the pseudorapidity $\Delta \eta$ of the negatively and positively-charged leptons:

$$
\cos \left(\theta_{\eta}^{*}\right) \equiv \tanh [(\Delta \eta) / 2] .
$$

Measurements are made of the normalised $\phi_{\eta}^{*}$ distributions in bins of the $Z$-boson rapidity for the dilepton invariant mass range of $66-116 \mathrm{GeV}$ using $4.6 \mathrm{fb}^{-1}$ of $p p$ collision data [4]. The normalised differential cross sections in both dielectron and dimuon final states are compared to Quantum Chromodymnamics (QCD) predictions such as those provided by the RESBOS [8-10] generator which resums the leading contributions up to next-to-nextto-leading logarithms and matches the result to fixed-order calculations in the strong coupling constant $\alpha_{s}$, corrected to $O\left(\alpha_{s}^{2}\right)$. Comparisons are also made to the predictions of various MC event generators implementing parton-shower algorithms. Figure 4 demonstrates the incredible resolving power of $\phi_{\eta}^{*}$ between generators and showering models. The difference between data and RESBOS is of order $2 \%$ for $\phi_{\eta}^{*}<0.1$ to as much as $5 \%$ as $\phi_{\eta}^{*}$ approaches unity. 


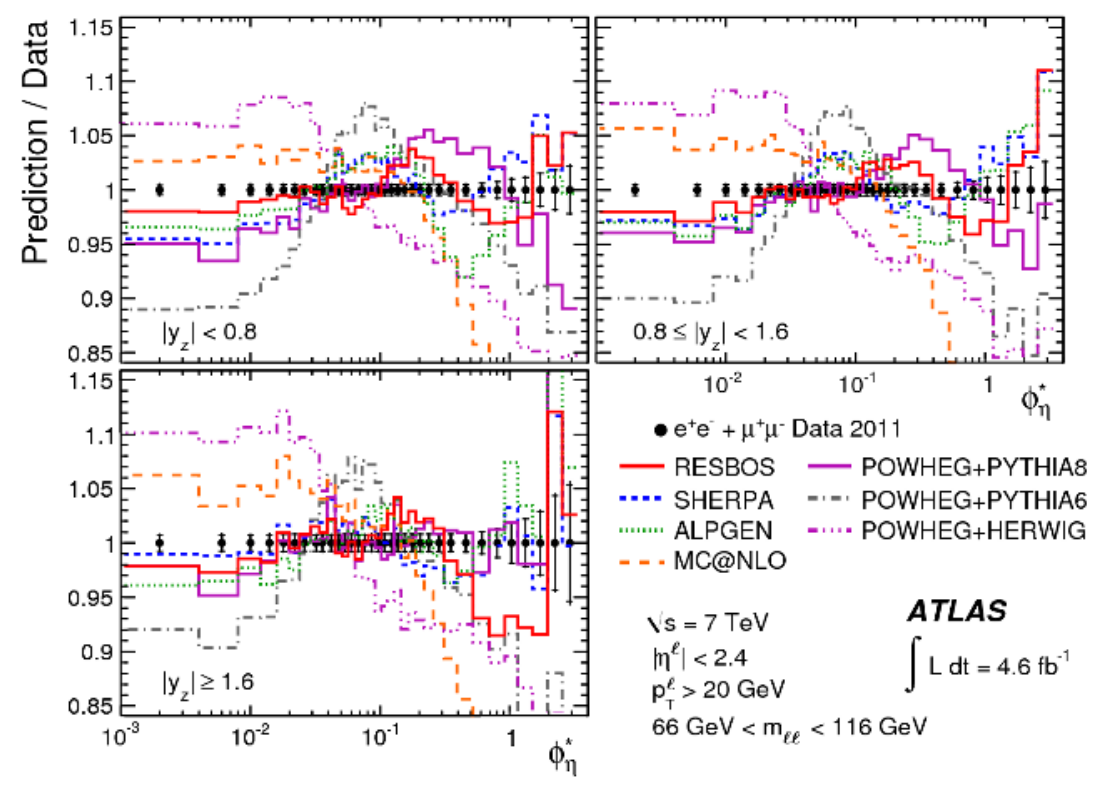

Figure 4. Ratios of different predictions to the measured differential cross section as a function of $\phi_{\eta}^{*}$ in three bins of Z-boson rapidity [4]. The measurements are compared to MC predictions implementing parton-shower algorithms, as given in the figure.

The best MC predictions are able to describe the data over the entire $\phi_{\eta}^{*}$ range to the $5 \%$ level.

\section{Conclusions}

Approximately three million Drell-Yan events decaying to electrons and muons have been recorded with the ATLAS detector in 2011 at $\sqrt{s}=7 \mathrm{TeV}$. Measurements based on these data have already provided rich information on the strong interaction and helped to constrain the parton distribution functions of the proton at LHC collision energies. The incredibly successful LHC running period of 2012 will provide a factor of four more integrated luminosity at an increased centre-of-mass of energy of $8 \mathrm{TeV}$. These data will be used for measurements that will provide insight of increasing precision and scope into electroweak and QCD physics.

\section{References}

[1] ATLAS Collaboration, JINST 3 S08003 (2008)

[2] ATLAS Collaboration, arXiv:1305.4192 [hep-ex], submitted to Phys. Lett. B (2013)

[3] ATLAS Collaboration, ATLAS-CONF-2013-043, https://cds.cern.ch/record/1544035

[4] ATLAS Collaboration, Phys. Lett. B720 32 (2013)

[5] ATLAS Collaboration, ATLAS-CONF-2013-017, https://cds.cern.ch/record/1525524

[6] J. C. Collins and D. E. Soper, Phys. Rev. D16 2219 (1977)

[7] Particle Data Group Collaboration, K. Nakamura et al., J. Phys. G37 075021 (2010)

[8] G. Ladinsky, C. Yuan, Phys. Rev. D50 4239 (1994)

[9] C. Balazs, C. Yuan, Phys. Rev. D56 5558 (1997)

[10] F. Landry et al., Phys. Rev. D67 073016 (2003) 